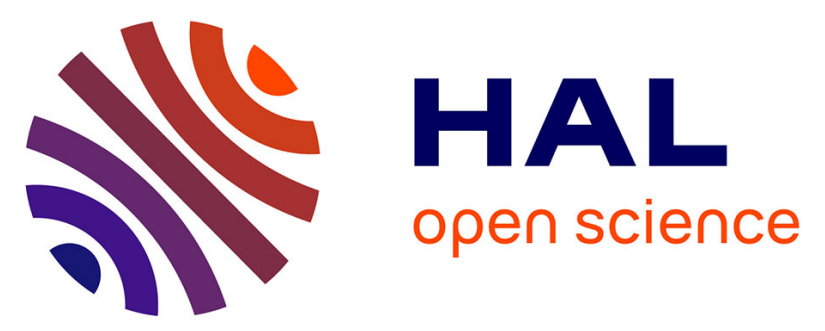

\title{
Refounding of Activity Concept? Towards a Federative Paradigm for Modeling and Simulation
}

\author{
Alexandre Muzy, Franck Varenne, Bernard P. Zeigler, Jonathan Caux, Patrick \\ Coquillard, Luc Touraille, Dominique Prunetti, Philippe Caillou, Olivier \\ Michel, David R.C. Hill
}

\section{To cite this version:}

Alexandre Muzy, Franck Varenne, Bernard P. Zeigler, Jonathan Caux, Patrick Coquillard, et al.. Refounding of Activity Concept? Towards a Federative Paradigm for Modeling and Simulation. SIMULATION: Transactions of The Society for Modeling and Simulation International, 2013, 89 (2), pp.156-177. 10.1177/0037549712457852 . hal-00738218

\section{HAL Id: hal-00738218 https://hal.science/hal-00738218}

Submitted on 3 Oct 2012

HAL is a multi-disciplinary open access archive for the deposit and dissemination of scientific research documents, whether they are published or not. The documents may come from teaching and research institutions in France or abroad, or from public or private research centers.
L'archive ouverte pluridisciplinaire HAL, est destinée au dépôt et à la diffusion de documents scientifiques de niveau recherche, publiés ou non, émanant des établissements d'enseignement et de recherche français ou étrangers, des laboratoires publics ou privés. 


\title{
Refounding of Activity Concept? Towards a Federative Paradigm for Modeling and Simulation
}

\author{
Alexandre Muzy ${ }^{1}$, Franck Varenne ${ }^{2}$, Bernard P. Zeigler ${ }^{3}$, \\ Jonathan Caux ${ }^{4}$, Patrick Coquillard ${ }^{5}$, Luc Touraille ${ }^{4}$, \\ Dominique Prunetti ${ }^{1}$, Philippe Caillou ${ }^{6}$, Olivier Michel ${ }^{7}$, \\ David RC Hill ${ }^{4}$. \\ Research Group on Activity Theory: Jonathan VAZILY
}

\footnotetext{
${ }^{1}$ LISA UMR CNRS 6240, Università di Corsica - Pasquale Paoli, 6, av. Jean Nicoli, BP 52, 20250 Corti - France.

${ }^{2}$ GEMASS UMR CNRS 8598 (Paris Sorbonne) - University of Rouen - UFR LSH,

Department of Philosophy, Rue Lavoisier, 76821 Mont-Saint-Aignan - France.

3 ACIMS (Arizona Center for Integrative Modeling and Simulation) - University of Arizona, Tucson, AZ - United-States of America.

${ }^{4}$ LIMOS UMR CNRS 6158, Blaise Pascal University BP 10125, AUBIERE Cedex - France.

5 Ecologie Comportementale et Moléculaire, Université de Nice - Sophia Antipolis, UMR

CNRS-INRA-UNSA 6243, 400 route de Chappes, P.O. box 167, 06903, Sophia-Antipolis, France.

${ }^{6}$ Laboratoire de Recherche en Informatique (LRI), INRIA - TAO Project, Université Paris Sud XI - IUT de Sceaux.

${ }^{7}$ LACL - Bât P2 - 240, Université de Paris XII, Faculté des Sciences et Technologies, 61 Av. du Général de Gaulle, 94010 Créteil Cedex - France.
}

\begin{abstract}
Currently, the widely used notion of activity is increasingly present in computer science. However, because this notion is used in specific contexts, it becomes vague. Here, the notion of activity is scrutinized in various contexts and, accordingly, put in perspective. It is discussed through four scientic disciplines: computer science, biology, economics, and epistemology. The definition of activity usually used in simulation is extended to new qualitative and quantitative definitions. In computer science, biology, and economics disciplines, the new simulation activity definition is first applied critically. Then, activity is discussed generally. In epistemology, activity is discussed, in a prospective way, as a possible framework in models of human beliefs and knowledge.
\end{abstract}




\section{Introduction}

In computer science, as pointed out in [1], the notion of activity is a very generic term, which can be applied to a variety of different topics. This notion of activity is different from the classical notion used in simulation. The usual activity notion can be found in Tocher [2], who also first described the three phases approach, as an optimization of an activity-based simulation. In [3], Balci presents the concept of activity as a possible approach to drive the implementation of a discrete event simulation kernel. An object-oriented variant of the three phases approach was introduced by Pidd [4].

In many fields, the notion of activity can be found. For example, it is a fundamental issue in computer graphics, from Z-buffers [5], to current work required for fast rendering of different level of details [6, 7] in complex scenes or multiresolution modeling in game engine [8]. In autonomic systems [9, 10], ensuring the persistence of the self- $*$ properties requires a feedback loop based on tracking certain variables that account for activity changes in the system, from the level of the operating system (e.g., in Solaris 10) to the level of large cloudbased systems. In everyware/ambiant/pervasive/ubiquitous systems [11], the key issue is to track the activity/location of a user to adapt local devices to the presence/absence and movement of the user's activity. Nowadays, any parallel system copes with dynamic requirements for resources using load-balancing [12] algorithms to track the activity taking place in each computing site to reallocate and reschedule tasks according to changes in both the demands and the availability of resources.

When modelling and simulating dynamic systems, the notion of activity is a key notion, which, in some contexts, can lead to structure changes of the state space as coined by [13], with the notion of dynamic systems embedding a dynamic structure. An attempt to quantify and formalize a simulated system activity has been proposed in [14] for model exploration.

Even if many computer science studies attempt to frame the notion of activity for modelling and simulating systems, this task remains interdisciplinary. Therefore, to extend the definition of activity, looking in the mirror of other disciplines would be judicious. Although the concept of activity is found in many disciplines, very few address activity explicitly. Activity concept is used idiosyncratically. Analyzing activity in every discipline, in the same study, should allow federating this notion and building more abstracted elements. Finally, it is expected that interactions between disciplines will be facilitated.

This study aims at using the activity notion through four scientific disciplines: computer science, biology, economics, and epistemology. Our goal is to analyze activity answering in every section/discipline the five fundamental questions:

(i) What is activity in this context?, (ii) Why use it?, (iii) How to define it?, (iv) Where to use it?, (v) When to use it?

It is believed that: activity can be used at many specification levels, in a federative interdisciplinary approach, to achieve an activity-based architecture for 
modeling and simulating component systems.

If we come back to the definition of the word Activity, we can realize that it is derived:

"From Latin: actives. Meaning 'state of being active, briskness, liveliness' recorded from 1520s. A specific deed, action, function, or sphere of action: social activities - A work, esp. in elementary grades at school, that involves direct experience by the student rather than textbook study - An energetic activity; animation; liveliness - A use of energy or force; an active movement or operation - A normal mental or bodily power, function, or process - An organizational unit or the function it performs" (American Heritage@) Dictionary.)

This mille-feuille meaning can be refined progressively. To approach, draw, and catch the fundamental entities of activity, a first attempt should be discursive.

First, a brief state-of-the-art of activity in Simulation and then a new definition is proposed ( $c f$. Section 2). In Computer Science, cellular interactions and their ability to exhibit life by reproduction has been majestically modeled by John Von Neumann and his Theory of Self-Reproducing Automata [15]. In Section 3, activity definitions of Section 2 are applied in the context of Game of Life artificial metaphor. "Artificial interactions" are investigated. Then, "biological and ecological interactions" are considered, modeled, and simulated through activity $^{1}$ in Section 4. After the study of both artificial and biological interactions, activity is considered in decision processes achieved by men living in society from an economics point of view ( $c f$. Section 5). Finally, the reflexivity of the modeling process through the use of activity definitions is presented. By reflexivity we mean the modeling mechanisms achieved by the subject with respect to knowledge and beliefs. The broad sense of modeling (including the decision process) is thus re-considered in epistemology (cf. Section 6) as a research perspective.

\section{Activity in Discrete-Event Systems}

We re-introduce here the definitions of activity proposed in [16].

\subsection{Previous Definitions in Simulation}

Balci described the four major frameworks that were in use to implement discreteevent simulation kernels [3]. These conceptual frameworks (also named simulation structures or simulation strategies or world views) guide scientists in the design and the development of their simulation model. In this paper we confer a particular attention to the activity scanning approach and to the activity

\footnotetext{
${ }^{1}$ Approaching the energetic aspects contained in activity, correlated to the notion of forces and movements initiated by a source of energy.
} 
concept itself. Activity scanning is also called a two-phase approach, the first phase being dedicated to simulation time management, the second phase to the execution of conditional activities (e.g., during scanning, execution of simulation functions depend on the fulfillment of specific conditions). In artificial intelligence this approach is known as rule-based programming (also known as rule-based systems or expert systems) [17]. Buxton and Laski introduced this approach in the simulation field with the Control and Simulation Language (CSL) [18]. In CSL, when a rule is "fired" a corresponding action is taken and the system state is updated. This approach is often considered to be dual with the event scheduling method. The fourth conceptual framework is named the three phase approach. It is an optimization of the activity scanning approach proposed by Tocher a year after the introduction of CSL [2]. This optimization is interesting for systems in which potential activities can be detected at each time step. The first phase is the same as in the activity scanning approach. The second phase is different since it handles the execution of all unconditional activities (avoiding rules scanning for rules known to always be fired). The third phase is then similar to the second phase of regular activity scanning (an activity is considered and executed if the corresponding rule can be fired). Pidd [4] provides more details on the latter approaches. Both activity scanning and three phase approach, as described by the literature, rely on a fixed time increment. The whole simulation is driven by clock time advance. This time management, often called clock-based by simulation practitioners, is also named continuous time by theoreticians $[19,20]$. Zeigler considers the fixed timed management as a discretization of a continuous time function. Because of data structure management, this approach can be inefficient when you get a lot of discreteevent occurrences at the same time, without detecting activities. In addition, it can also lead to inaccuracies when high precision simulations are considered. An event-based time management where the time is advanced to the next scheduled event avoids the two previously cited problems.

Let's review the previous definitions [mainly extracted from [3]] of the following words : activity, event, and process, which are the underlying concepts in this paper. An activity is an operation that transforms the state of a system over time. It begins with an event and ends by producing another event (linked to the termination of the activity). Some definitions in the simulation community consider that an activity is thus a period of time with a known duration, constant or random, computed or read in a file if we have a trace based simulation. An event is what causes a change in the state of the system (eventually composed of many components). A process is a sequence of activities or events ordered in time. A process is usually linked to the object, actor or agent oriented approach in which the life cycle of an active entity is specified. As it can be seen, events (underlined in previous definitions) are central for usual world views. We propose here a new fine-grain definition of activity grounded on discrete-events. Then, a full activity-based modeling and simulation life cycle is presented. 


\subsection{A New Definition of Activity}

In the previous definitions, activity emerges as a quality of objects. Traditionally, activity, as a measure (a quantity), has been scarcely used at the implementation level [14], and not at all at the conceptual level (except some recent exceptions we will see). Nonetheless, in all the previous definitions presented in introduction, discrete-events (underlined) are central - as countable units. We propose here a new integrative (qualitative and quantitative) definition of activity. First, since events occur as a consequence of system activities, we can consider that:

Definition 1. Qualitative activity in a discrete-event system: A system is qualitatively inactive when no events occur and qualitatively active otherwise.

Second, at the beginning of the nineties, a $\mathrm{C}++$ simulation library, named Meijin ++ , proposed an interesting implementation of a discrete-event simulation kernel. It proposed a dynamic processing of events with different data structures selecting at runtime the best data structure depending on the number of events, or their frequency of occurrence and on the overhead needed to copy data from one structure to the other. We consider here that:

Definition 2. Quantitative activity in a discrete-event system: The sum of both internal quantitative activity and external quantitative activity is equal to quantitative activity, over a simulation time period. Discrete-events can be of two types: internal or external to the atomic model (endogenous or exogenous). Internal quantitative activity corresponds to the number of internal discreteevents, over a simulation time period. Internal activity provides information about the quantity of internal computations within atomic models. Quantitative external activity corresponds to the number of external discrete-events, over a simulation time period. External activity provides information about the quantity of messages exchanged by atomic models.

Measuring quantitative activity has been used with success for the simulation of spatially distributed systems in [21]. Notice a crucial distinction, quantitative activity is defined at the simulator level and is a metric of computational resource usage, while qualitative activity is defined at the model level although it can be used at the simulator level.

Having proposed a new definition of activity, we now want to know how activity can be used in the usual model and simulator architecture of DEVS. First, considering qualitative activity, atomic models can be defined as embedding a binary state variable: qualitativeActivity $=\{$ active, inactive $\}$. This has been widely used in DEVS [cf., e.g., [22, 23]], where cells can be active or inactive]. At each time step, knowing that some atomic models are active and others are inactive, simulators can focus computations only on active atomic models [24]. This is the activity tracking mechanism. Hence, activity tracking is defined as 
the ability of simulators to automatically detect active atomic models, focusing simulation resources only on these atomic models, during a simulation run.

Cellular automata are a good application case for activity tracking. When simulating cellular automata, the Hash-Life algorithm, recently re-introduced in the Golly software (http://golly.sourceforge.net/), is a very good example of activity tracking. Spatial patterns of qualitative activity are detected at various sizes, from the well known elementary patterns to complex meta-cells. A memorization optimization, more classically used in recursive algorithms, uses a hash table, where discovered patterns are directly linked to their simulated future thus avoiding unnecessary re-computation. This activity tracking strategy enables to simulate huge cell spaces (above $10^{50}$ cells) on a regular personal computer.

\section{Determination of Activity Configurations in Cel- lular Automata}

Considering wave propagations in space, through cellular automata ${ }^{2}$, constitutes a direct analogy with activity propagation paths. Activity is located in space where the wave propagates. Inactivity is located in space where the wave does not propagate. When building the corresponding simulator, computational resources can be allocated dynamically to track activity. The allocation of simulator resources depends on "how sparse" is the activity. Categories of activity propagations can be classified between two extrema: (i) a large wave spreads, at the same time, in every site of the space, computations need to be achieved in every site, without accounting for activity tracking (which would be inefficient.), (ii) a single thin wave smoothly propagates in a huge space; activity tracking exhibits very good performances.

During a propagation, activity in space can be determined quantitatively and qualitatively. At the lowest level, basic activity corresponds to a simple binary state of cells. At the highest level, activity configurations can be identified to build an activity map of activity propagation. Elements of the map correspond to well defined reproductive and re-occurring configurations. The whole activity level can be quantitatively determined as a percentage of active cells in space. Quantifying and tracking activity configurations and simple active cell change states is described hereafter.

\footnotetext{
${ }^{2}$ Standard cellular automata consist of an infinite lattice of discrete identical sites, each site taking on a finite selection of, for instance, integer values. The values of the sites evolve synchronously in discrete time steps according to deterministic rules that specify the value of each site in terms of the values of neighboring sites. CA are models where space, time and states are discrete.
} 


\subsection{Simulation in an Excitable Medium}

The simulation of wave propagation can be implemented combining existing efficient algorithms and an activity-based description of the system.

\subsubsection{Excitable Medium}

The cellular automata considered in this paper are applied to an excitable medium. An excitable medium is a nonlinear dynamic system that can propagate a wave. Medium areas that have just propagated a wave lose their propagation capacity for a given period, called refractory period.

Activity-based states can be used to build dynamically a set of active cells in which computations occur, and, conversely, computations do not concern inactive cells [25]. Here, the cellular automaton uses a 3D von Neumann neighborhood with three major states:

1. activated state: a cell remains in this state for a predefined amount of time.

2. refractory state: a cell evolves to this state after spending a predefined period of time in the activated state. In this state, a cell cannot be excited by an activated neighboring cell. A cell remains in this state for an amount of predefined time.

3. inactive state: a cell remains in this state until a neighboring cell turns into the activated state; in which case, the cell becomes activated.

For example, these three activity-based states can be used to roughly model the electrical stimulation of heart cells. Depolarizing heart cells can be represented as activated cells. Repolarizing heart cells can be represented as refractory cells. Finally, other heart cells states can be represented as inactive cells. A more precise model of the heart electric stimulation can be found in [26].

\subsubsection{Background on Hashlife}

Hash Life [27] is a simple, yet powerful, algorithmic technique, which allows the simulation of very large cellular automata using memoization. Memoization consists in avoiding the repetition of the same computations. Computation results are progressively stored in memory. The algorithm stores for every configuration of cells the result of the computation of the next iteration. However, to be efficient, this optimization requires the cells configurations already encountered to be accessed easily. Two mechanisms can be used to achieve this goal: (i) An octree for 3D representation (quadtree for 2D representation), and (ii) A hash table for the configurations of cells storage. 
An octree is a tree data structure [28] where each node is either a branch which can have up to eight child nodes, or a leaf. In the case of Hashlife, a complete octree is used: each branch has exactly eight children. Each node does not store its children instances but only their memory addresses. It allows having only one instance of each cells configuration which is shared between many nodes. In Figure 1, a branch has its eight children composed of the same cells configuration. Thus, this branch has only eight pointers to the address of the same instance (@b). The comparison of cells configurations can be achieved simply by comparing their eight children memory addresses.

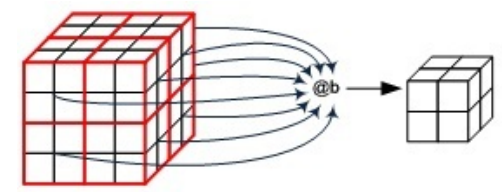

Figure 1: Example of Multiple Aggregations of the Same Branch Instance.

In the hash table, the key corresponds to the eight children memory addresses and the value corresponds to the instances of the corresponding cells configurations. Knowing the eight children composing a configuration, it is possible to access the corresponding instance in the map. Because the result of the next iteration is stored with the instance, if it has been already computed, it is also accessed at the same time. Obviously, if it is the first time that the cells configuration is encountered, it will not be present in the hash table; this configuration should be added to the hash table and its next iteration computed.

\subsection{Limitations of the Activity Definition at the Cells Level}

To fully understand how this algorithm accounts for activity, it is important to clearly determine what is the activity in this context. Thus, the next part will try to apprehend this concept and formulate a definition of activity allowing a better understanding of the algorithm performances.

\subsubsection{A First Activity Definition for Excitable Medium}

A first level definition can be established:

\section{Activity Hypothesis 1: Cells activity}

If qualitative activity corresponds to a state change in a cell, then, in a memoized cellular automaton, activity is defined at the cells level, and, intuitively the more cells change state, the more quantitative activity increases and then the more computation overhead increases. 
To verify this hypothesis, two simulations have been run in excitable medium with various parameters. The simulation of wave propagation takes place in a cube of $10243^{3}$ cells.

In the first simulation, new waves appear randomly according to an exponential law distribution of rate parameter $\lambda=0.1$ (one stimulation every 10 time step on average). These waves appear randomly on the entire cube. In the second simulation, new waves appear according to an exponential law distribution of rate parameter $\lambda=0.125$ (one stimulation every 8 time step on average). Waves appear on a corner.

Figure 2 represents the percentage of active cells on the entire cube. Logically, the second simulation, which has more waves initiated, has rapidly more activity on the entire cube than the first simulation. If Activity hypothesis 1 were correct, the execution time of the second simulation should become larger at each time step (cf. Figure 3), as soon as the activity of simulation 2 becomes greater than the activity of simulation 1 .

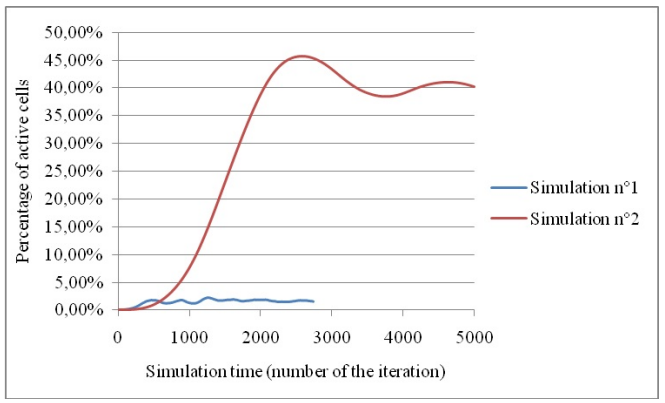

Figure 2: Percentage of Qualitative Activity on the Entire Cube at Each Time Step.

Quantitative activity of cells has no direct impact on simulation performances. Although there are seven times less activity at the same time in simulation 1, slowest time step computations are about 15 times slower than in simulation 2. In simulation 2, one computation is always computed in less than $0.03 \mathrm{~s}$ whereas in simulation 1 computations are completed up to $0.4 \mathrm{~s}$.

These results demonstrate that a large number of active cells does not involve necessarily a large execution time. Indeed, this is due to a difference in the quantity of configurations between simulation 1 and simulation 2. There are less dissimilar configurations of cells generated in the second simulation. In the first simulation, because waves are initiated in many points in the cube, a lot of new configurations of cells occur.

As depicted in the example of Figure 4, even if simulation 1 has less cells activated, there will be more different configurations of cells than in the second simulation where all the wave are very similar. Therefore, since Hashlife uses 


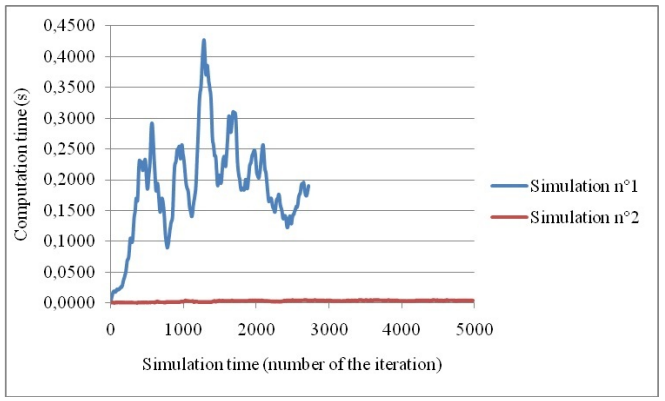

Figure 3: Execution Time of Each Time Step.

memoization, the execution time cannot be only related to cells activity, another definition of activity needs to be explored.
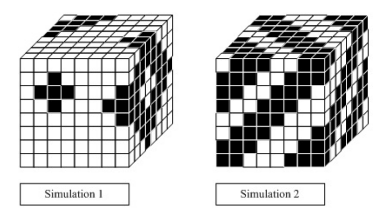

Figure 4: Examples of Simulations Configurations.

\section{Activity Hypothesis 1 is false:}

If qualitative activity corresponds to a state change in a cell and activity is defined at the cells level, then, in a memoized cellular automaton, an increasing number of state changes in cells does not involve necessarily a computational overhead.

\subsubsection{Activity Definition at the Cells Configuration Level}

\section{Activity Hypothesis 2: Activity Configurations}

If qualitative activity corresponds to a state change in a cell and inactivity corresponds to no state change in a cell, then, in a memoized cellular automaton, a configuration consists of a set of active and inactive cells and an increasing number of configurations involves necessarily a computational overhead.

For example, the three configurations of Figure 5, which have different numbers of active cells, will be computed in the same way by Hashlife. A configuration with less active cells (like the one with only inactive cells, in configuration 1) does not require less computation time than other configurations 2 and 3 , with more active cells. 


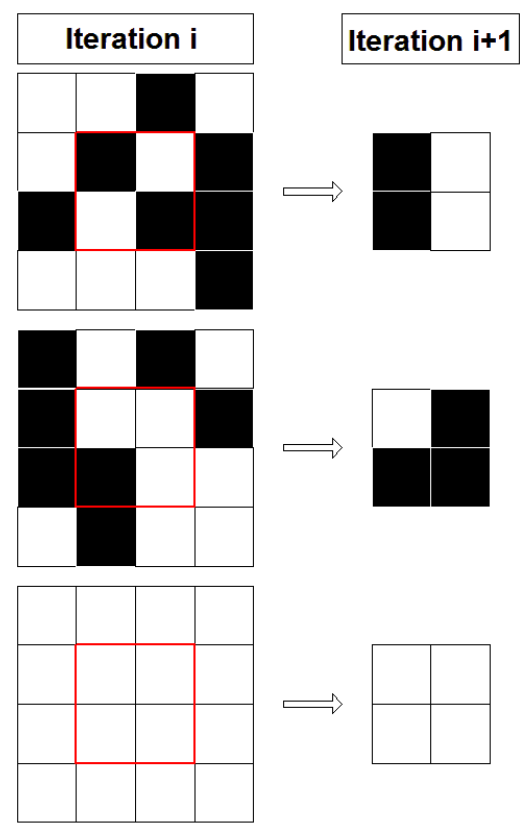

Figure 5: Examples of Configurations of Cells. 


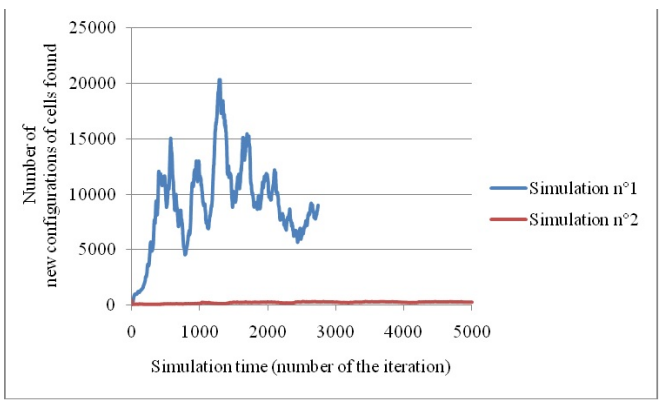

Figure 6: Number of New Configurations at Each Time Step.

\subsubsection{Activity Definition based on Model and Algorithm}

The definition and tracking of activity configurations is required to implement efficiently Hashlife. Model activity consists of the number of new configurations of cells. Here, the number of cells changing state is not the main factor of activity:

- a simulation with a high cellular activity can have little global quantitative activity if the same configurations of cells re-appear regularly;

- conversely, a simulation with less cell activity can exhibit a higher global quantitative activity if the cells changing state evolve rapidly to various configurations.

Figure 6 shows the number of new configurations of cells found at each computation in both simulations. When compared to Figure 3, we figure out that the number of activity configurations is strongly related to the execution time required by Hashlife. In simulation 1, even if the number of cells in which the number of state changes is lower than for simulation 2 (see Figure 2), supplementary new configurations occur thus requiring more computations.

\section{Activity Hypothesis 2 is partially true:}

If qualitative activity corresponds to a state change in a cell and inactivity corresponds to no state change in a cell,

and If, in a memoized cellular automaton, a configuration consists of a set of active and inactive cells,

Then an increasing number of configurations can involve a computational overhead, depending on the activity tracking algorithm.

\subsection{To Sum Up}

A first attempt of activity definition in cellular models has been provided. Topological patterns (configurations) of both active and inactive cells proved to com- 
plete a basic cells activity definition. Configurations definition opens new research perspectives for abstracting multi-level configurations. For example, in the fractal propagation ( $c f$. Figure 7), a fractal configuration can be detected at many levels of details.

Two definitions have been experimented through memoized cellular automata and can be generalized to cellular automata. Considering this extension as a postulate, the two activity hypotheses are obtained:

\section{Activity definition 1: Activity in Cells}

In a cellular automaton, qualitative activity corresponds to a state change in a cell and inactivity corresponds to no state change in a cell.

\section{Activity definition 2: Activity Configurations}

In a cellular automaton, a configuration consists of a set of qualitatively active and inactive cells.

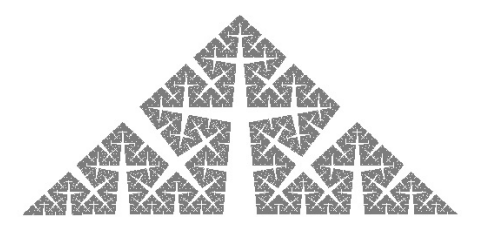

Figure 7: Fractal Configurations.

A possible issue of this activity definition is the difficulty to estimate beforehand the level of activity of a model. Nothing guarantees that fewer possible cell states involve fewer different configurations during the simulation. The only simple way to be sure of a model activity is to simulate it, and to detect it using ad hoc algorithms.

Concerning cellular automata implemented through Hashlife:

- What is activity in this context? An activity configuration consists of a configuration of active and inactive cells.

- Why use it? Focusing on occurrence of new patterns allows reducing the number of computations in models where new occurrences are not so frequent.

- How to define it? It is the frequency of occurrence of new configurations of cells which have not been already computed.

- Where to use it? In the implementation of simulators of cellular automata models.

- When to use it? When tracking pattern activity enables better performances than classical methods (depends on the model simulated). 


\section{Activity in Living Systems}

Within the context of biology and ecology, the answer to the question of activity leads - at first glance-to this simple and definitive response: "activity is anything but death". Thus, any biological object can be considered as active if it is alive... But things are not so simple. Let us consider some sub-cellular parts such as mitochondria, ribosome, cell nucleus, endoplasmic reticulum... Is a mitochondria alive? No, a mitochondria is a cellular organelle; a structure which can produce some energetic compounds (ATP) using sugar and oxygen (respiration). It's a kind of machine dedicated to chemical energetic transformations. Is it active? Yes.

The notion of function also has to be considered. A biological system is the locus of a set of functions and, sometimes, the number of functions can be reduced, altered, in case of malfunctioning (nutrient deficiencies, pathologies...). In addition, some functions are involved into homeostasis ${ }^{3}$ which aims at maintaining other vital functions. Regarding functions, the "active" status could be linked to the ability to maintain at least one expected function. But maintaining alive is much more complicated.

Finally, the definition of activity in this context seems strongly connected to the dialectical concept of life and death. However, it is not our goal to attempt to give a definition of life (yet, scientists cannot agree on an absolute definition). Modeling and simulation of biological systems can lead to one of these two situations:

1. The system we are interested in is of sub-cellular type. In such conditions, the system is supposed to be encapsulated into one -or more- living cells. The structure "cell" is then considered alive as long as the system is active.

2. The system is composed of one or more cells. This system is active as long as one cell at least is alive, which does not mean that the system itself is still alive.

Hence, we can see that defining a biological system activity depends on the aggregation level at which the model is defined (sub-cellular, cellular, tissue, organ, organism, population...)

\subsection{Activity Definition}

Let us now focus on the concept of activity. What is the essence of biological activity? The chemical point of view can help: is declared active any biological object which is the locus of both exchanges (gas, liquids, nutrients, molecules...) and transformations (chemical transformations), which result in maintaining the

\footnotetext{
${ }^{3}$ Property of living systems, which regulate their internal environment and tend to maintain a stable milieu interieur (sensu Claude Bernard).
} 
integrity of the object. At this point the problem of time granularity arises. If one observes a biological object with a very small step of time, some (short) inactivity phases can appear. Thus, the object can be considered as inactive if and only if its inactivity is recorded on a large enough interval of time. From a wider point of view, the inactivity of a biological object is stated on a statistical basis.

Basically, biological objects are systems. In other words, their activity is correlated with the exchanges and transformations (of matter and energy) between the parts of the system and between the system and its environment. As a first result of these few considerations, we can say that the activity of a biological system consists in (i) tracking the internal/external exchanges and transformations (ii) verifying the integrity of the system (iii) then to draw a statistic. Finally, one can consider that the definition of biological activity we sketched above is rather consistent with the definition of the quantitative activity in a discreteevent system simulation: "the sum of both internal and external discrete-events over a simulation time period is equal to the quantitative activity of the system."

\subsection{Measuring and Quantifying Activity}

The activity measurement of a biological/ecological system is a real challenge. We all know that measurement influences the system under study, and this is particularly true for living systems. Among others, a standard method consists in measuring the $\mathrm{CO} 2 / \mathrm{O} 2$ gas exchanged between the system and the atmosphere. This is in fact an indirect measurement of the respiration/photosynthesisactivity ratio of the system. But this measurement is a global one which cannot describe accurately the relative activities of the system components.

One particular characteristic of biological and ecological systems is redundancy. Thanks to redundancy, some unpredictable (unknown) properties not directly traceable to the system components can appear throughout its functioning. This is generally called the "emergence property". Redundancy is double: structural redundancy (several identical structures execute the same function) and functional redundancy (the same function is executed by several and different structures). Here again we highlight the notion of function ${ }^{4}$, indeed, it immediately appears that if a function is executed (i.e. the system is active), one or more structures can be implicated. Thus, the measurement of a function by means of its production over time (concentration of metabolic compounds, number of descendants...) cannot be a reliable estimation of the activity. In other words it does not give information about what is active and what is inactive in the system.

\footnotetext{
${ }^{4}$ In biology, at a first glance, a behavior is the resultant of the execution of multiple functions.
} 


\subsubsection{Useless Activity}

This is a wider problem. In the context of modeling and simulation, the problem addressed could embed a lot of active processes, which can potentially participate at various levels to the response. In this case, it is essential to test which processes have significantly contributed to the response. Some statistical and efficient methods are available to reach this goal.

\subsubsection{Activity of the Whole}

Is a system active or inactive? Is its activity quantifiable? These are some crucial questions. Figure 8 depicts the variation of $\mathrm{CO} 2$ concentration (in ppm) at Mauna Loa (French Polynesia). The positive trend indicates the increase due to human activities. But we can see that there is an obvious seasonal variation. The variation corresponds to the active period (i.e. from spring to the end of summer) alternating with the inactive period of vegetation (autumn and winter). In some sense, the curve gives a measure of the system activity. The derivative (i.e. the slope) indicates the velocity at which the system evolves (accelerations and slowing down phases). Minima and maxima point out some short periods where the $\mathrm{C} 02$ fixation equals the respiration of ecosystems.

An analogy-based reasoning led us to consider the simple case of a cellular automaton (a bounded grid) which aims at simulating the dynamics of several populations competing for space. The rules that dictate the evolution of cells are probabilistic. The probability of changing state depends on the neighbourhood of the cell (be it a Von Neuman or a Moore or even a more exotic neighbourhood pattern), i.e. the number of individuals of each species and their state (age, reproduction potentiality and so on...) that surrounds the cell. Each cell can take one of a finite -and known- number of states (uncolonized, species 1, species 2, . . . , species $n$ ) and we attempt to evaluate the activity of the system through time.

Quantitative activity is defined as the recording of the number of each of the states $i, i \in[1 \ldots n]$, at each time step $t$ of the simulation: $n_{i}(t)$. This allows calculating, from collected data, the slopes $\left(V_{i}\right)$, as an approximation of the derivative: $V_{i}(t)=\frac{n_{i}(t+\Delta t)-n_{i}(t)}{\Delta t}$. The slope corresponds to the average rate of quantitative activity of the whole system. Then, for each time step we have $\sum_{i=1, ., n}\left(V_{i}\right)=0$ and $\sum_{i=1, ., n}\left(V_{i}\right)^{2} \geqslant 0$. Thus, the average rate of evolution (or speed) of the automaton is: $V(t)=\frac{1}{n} \sqrt{\sum_{i=1, . ., n}\left(V_{i}(t)\right)^{2}}$. However, even if $V(t)=0$, some modifications $M$ can persist and compensate each other. Thus, $M(t)=\frac{1}{n} \sum_{j=1, \ldots, E} P_{j}(t)$, where $P_{j}(t)$ is the entity $(j)$ modified at the instant $t$ and $E$ the overall number of entities. $M(t)$ is the average of qualitative activity changes of entities $N . M(t)=0$ in case of inactivity but is a constant $(>0)$ in case of equilibrium. Since $V(t)$ and $M(t)$ are in same dimension, the activity function $A(t)$ of the system takes the form: 


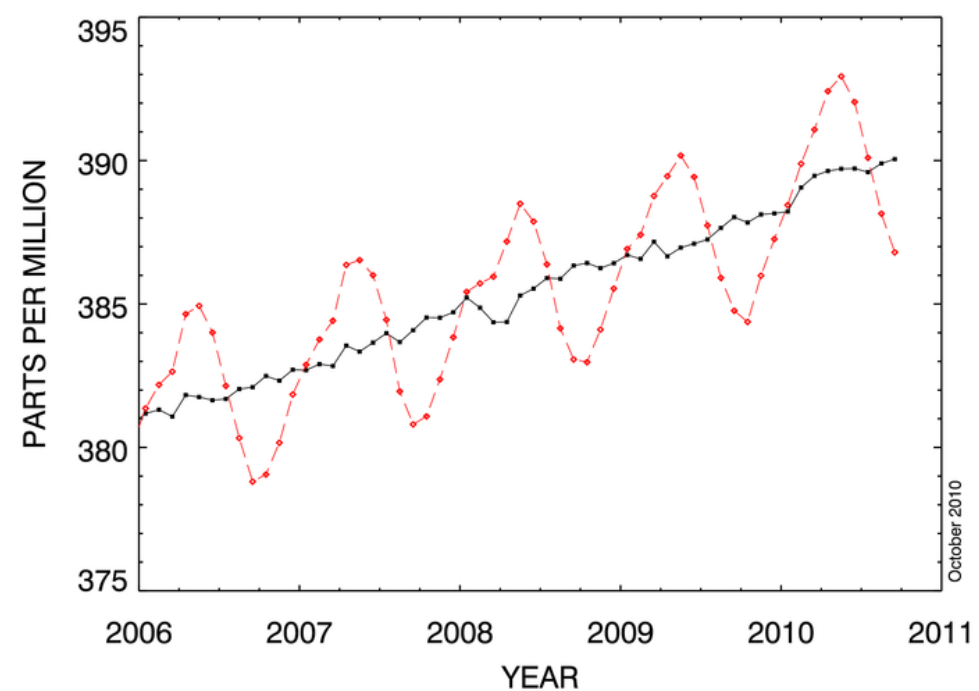

Figure 8: Monthly Mean of CO2 Concentration (in ppm) at Mauna Loa (Hawaï). The dashed line represents the monthly values. The solid line represents the seasonally corrected data (moving average of 7 adjacent seasonal cycles centred on the month to be corrected) showing a positive trend attributed to the increasing human activities[29]. 


$$
A(t)=V(t)+M(t)=\frac{1}{n}\left(\sqrt{\sum_{i=1, . ., n}\left(V_{i}(t)\right)^{2}}+\sum_{j=1, . ., E} P_{j}(t)\right)
$$
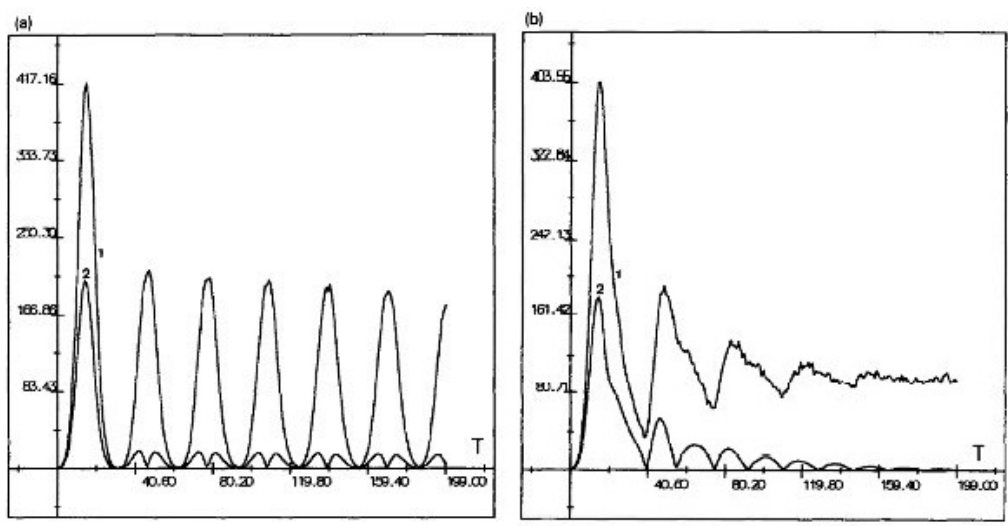

Figure 9: Activity Function of a 3 States Cellular Automaton through Time[30]. "1" $=M(t)$, " $2 "=V(t)$. (a) Using a high rate of reproduction of one of the 3 states, the system presents a cyclic activity (quasi stationary equilibrium) with an alternation between intense activity periods and short inactivity periods. (b) Using a low rate of reproduction (i.e., the highest probability to reproduce for one of the 3 species into a free adjacent cell is below a threshold value), the system converges to an equilibrium state in which $V(t)$ is null but $M(t)$ shows that the system is still active.

However, this simple proposal suffers from the lack of defining accurately what a "change of state" is. Change of state could take many forms, discrete changes or continuous as well (a variation of energy level for instance). May be the latter statement constitutes a crucial point to go deeper in the reflection about what activity signifies, and how the concept could help in saving computing resources and get (i) a better description of the dynamics, (ii) a better appreciation of the potentialities of the systems under study.

- What is activity in this context? Activity can have different meanings depending on the aggregation level at which the system is considered.

- Why use it? To determine which components of a system (cell, individual, population, ecosystem...) participate in the achievement of a particular task (or behaviour) and in some cases which components are essential to maintain the integrity of the system. In some cases, the prediction of the activity level over time of a biological system (organism, ecosystem...) can help in optimizing some actions (therapies, ecological engineering and 
management...) The prediction of the activity variation over time of simulated biological systems (set of functions, organism, ecosystem...) can help in optimizing some actions (therapies: optimal frequency/dose of medication impulses; ecological engineering and management: best date (or state of a system) to engage actions (modifications, treatments, introduction of species, etc.)

- How to define it? Today, no simple definition can be given in this context. However, we make this proposal, as a basis for further studies: is declared qualitatively active any biological object which is the locus of exchanges (gas, liquids, nutrients, molecules...) and of transformations (chemical transformations) which result in maintaining the integrity of the object itself or the system to which it belongs.

- Where to use it? In experimental biology and ecology and within the prediction tasks of the system behaviour (modeling and simulation).

- When to use it? In decision making (experiments and modeling) and to improve the effects of some actions on systems. Crucial in the analysis of system functioning. For instance, which genes involved in a network (socalled pathway) are activated/inhibited as a response to a (fluctuating) signal coming from the environment of the system under study?

\section{Activity in Economics and Decisions}

Activity is a central notion in Economics. Therefore, before re-defining or using a concept of activity, we need first to review what is economical activity and how economical activity is investigated in the framework of economic theories. In a second part, computational activity is modeled through optimal control theory. Optimal control is used in Economics to mathematically model and find optimal decisions. Finally, a notion of activity, compatible with both Economics and Computer Science, is proposed for activity evaluation.

\subsection{Economical Activity}

Following [31]: "Economics is the science which studies how scarce resources are employed for the satisfaction of the needs of men living in society: on the one hand, it is interested in the essential operations of production, distribution and consumption of goods, and on the other hand, in the institutions and activities whose object is to facilitate these operations".

Economics is interested by the satisfaction of the needs of men living in society in combination with scarce resources. Indeed, all studies in economics begin by the observation of the fact that human needs are infinite while resources are limited. The confrontation of these two observations involves compatibility 
problems and so decision problems: there is a need for allocation's decision rules at an individual level (allocation of budget for consumption or choice of input quantities for production for example) or at a collective level (street lighting or national defense for example).

According to this definition, economics is concerned by all human "activities", consumption or production problems, but also by all problems involving human activities like, for example, allocation of time to tooth-brushing. The economic approach to human behaviour by [32] has even extended economics' fields to questions traditionally covered by the sociology of the family economy and marriage market economy policy, economics of education, economics of altruism, suicide, religious attitudes... In fact, according to Becker and his followers, economics should not be distinguished from the other disciplines, but should be seen as a method that would be the only one able to provide a unified explanation of a wide range of human activities.

In this sense, an economic activity is a phenomenon impacting a scarce resource. The impact of this activity can be measured individually by economic agents' utilities.

Considering this definition (activity is what economic actors do, utility is usually the result of an economic activity), the notion of economic activity has been considered differently by economic fields:

- In the mainstream neoclassical economics, the focus is not on actors activities, but on their result (profit and utility).

- In behavioral economics, the focus is on activity selection (what actors do), not on utility anymore. Some models, like Rule Rationality, tentatively introduce maximization back into activity selection.

\subsection{1 (Neo)classical Economics and the Concept of Rationality}

Activity is a "core underlying" concept of every classical economic study: a core concept because most human activities are studied by economic theories, using several rationality models to determine how activities are selected. But also an underlying concept because economists mostly consider equilibrium situations computed with agents' utilities. They consider the activities consequences (the utility of the resulting situation) not the path (the activities themselves).

The rationality of an agent determines how he decides what to do concerning economic activities. [33] distinguished between two kinds of rationality that can be bounded:

- The substantive rationality: "behavior is substantively rational when it is appropriate to the achievement of given goals within the limits imposed by given conditions and constraints" [33] . The orthodox economists, from neoclassical economics, adopt the assumption that agents decision making is based upon substantive rationality. 
- The procedural rationality: "behavior is procedurally rational when it is the outcome of appropriate deliberation. Its procedural rationality depends on the process that generated it" [33] . Behavioral rationality corresponds to the decision process itself, not to the data used by this process (substantial rationality). Bounded behavioral rationality is not an optimization process anymore. This kind of rationality can be viewed as an alternative assumption to substantive rationality, but it has never supplanted it in the mainstream economists.

However, none of these rationality models consider activities themselves. Both consider alternative "utility bags", and eventually the process to select the best bag (procedural rationality). Rule rationality is one way to bring activity back into the economic agent decision process.

\subsubsection{Behavioral Economics and Rule Rationality}

Whereas neoclassical economics focuses on the economic activities results (the utility), behaviroral economics priorities are the opposite: its main problem is how actors choose their activities, how they choose what to do. One of the forerunners in behavoral economics, Robert Aumann, winner of the Nobel prize, proposed a form of bounded behavioral rationality called rule rationality by opposition to the traditional "Act rationality". "Ordinary rationality means that when making a decision, economic agents choose an act that yields maximum utility among all acts available in that situation; to avoid confusion, we henceforth call this act-rationality. In contrast, under rule rationality people do not maximize over acts. Rather, they adopt rules, or modes of behavior, that maximize some measure of total or average or expected utility, take over all decision situations to which that rule applies; then, when making a decision, they choose an act that accords with the rule they have adopted." [34]. The economic actor progressively learns what the best rules for different configurations are, and when he has to make a decision, he does not choose the best action, but the best rule in the current configuration, and applies it.

Aumann gives many examples of this model, for example the Ultimatum game without iteration. In the Ultimatum game, at each stage, player $A$ decides how to divide 100 Euros between himself and player $B$, for example 70/30. If player $B$ accepts, the repartition is made accordingly. If player $B$ rejects the proposition, no one gets anything. If agents are perfectly rational (in the sense of substantive rationality), A knows that for the last nth game, $B$ should accept any repartition, because 1 Euro is better than nothing. And recursively $A$ deduces that for the $(n-1)$ th game, the $(n-2) t h, \ldots$ the reasoning will be the same. And $B$ should accept.

Experiments show that when the Ultimatum game is played only once, without any knowledge or any contact with the other player, player $B$ will apply a threshold around 20. Explanations based on pride or threat do not fit anymore, since player $B$ does not know, see, or have any contact with its opponent 
and plays only once. But Rule rationality gives an explanation: Player $B$ has learned that in a general case, it is not good to accept too bad offers, both for reputational and threat reasons. And even if these rules do not apply here, they usually apply. So because in real world it would be act-rational to do so, it is here rule-rational to refuse a $90 / 10$ offer.

Another example concerns an experiment with bees and flowers. Bees behavior is studied in a field of artificial flowers with two colors: blue and yellow. An artificial mechanism allows choosing which flowers gives nectar. At the beginning of the experiment, only blue flowers give nectar. Bees begin to explore the field and quickly visit only blue flowers to get nectar. After some time, the blue flowers stop giving nectar and yellow flowers start giving some. The bees continue to go only to the blue flowers and finally die from starvation. The explanation of Aumann is that this kind of situation is not possible in nature. Bees have learned by evolution that it is interesting to recognize the flowers that give nectar and to stick to them because it is efficient. When they are put in the experimental environment they continue to apply this rule (stick to the identified color), they are rule-rational, even if this is not the best choice in this situation. Evolutionary learning has the same role as experience for human learning.

The interesting point about rule-rationality is that agents reason about activity (the rules activation), not about facts. They choose their action, not their consequences (the optimal situation). They are activity-aware, not situationaware anymore. Moreover, past activities determine current activities (via rulelearning).

\subsubsection{Economical Activity Evaluation}

To summarize, activity is an underlying concept in mainstream economics, but progressively emerges as a more central concept in behavioral economic studies such as Rule rationality. However, even in behavioral economics, the concept of activity itself is very rare and has to be deduced from the model. The focus is on activity selection, not on activities themselves. For this reason, to measure activity is still a challenge not considered by economists.

An evaluation of economic activity would have to be different from the main and well-studied indicator utility: if an agents eats three donuts, its utility is decreasing for each donut (it can even be negative for the last one - this is the decreasing marginal utility hypothesis), but the activity volume should be roughly the same. Another problem is that a precise evaluation of such economic activity would require describing it precisely, and we reach here the limits of the economic field as a science: Economics considers actors (humans or institutions) as "black boxes", with inputs and outputs. Describing the activity (and not only its selection process like in behavioral economics) would require to enter the black boxes, which are management (for institutions) or psychology 
(for humans) application fields. This field delimitation may be an explanation for the current absence of activity as a core concept in economic theory.

The genericity of component-based simulation approach may indeed bring some interesting and new results when applied to economic models. Quantitative activity as the number of discrete events received by the system is well suited to economic field since it does not require the description of the activity itself. It requires however discrete events, whereas classical economics considers equilibrium and continuous functions (atomicity hypothesis). Translated into the economic field and vocabulary, activity would be the number of events concerning scarce ressources (consumption, production, transaction, ...). It can be used to describe where "something" happens and when. Classical economics describe the result. Behavioral economics explains why, activity analysis could describe what happens (where and when). More importantly, it could describe what happens, the spaciality and the dynamicity of the system, without entering the black box. For example, let us consider a city-wide economic transaction analysis. Classical economics would give the global welfare and its repartition between the actors (people and firms). Behavioral economics would explain why people would buy such item and accept to pay such prices. An activity analysis would give us the repartition of the events in the city and its evolution. An observer could know where most of the production/consumption/transactions occur and when. Differenciation between external and internal activities depends on analyzed subject. If the focus is a city, any event occuring inside the city increases internal activity, whereas events relating to other cities (such as population or production transfer) are external activity.

\subsection{Optimal Control Model of Activity}

Here we consider the following problem: A modeler is designing a simulator for solving some model. As in every modeling approach, his main goal is to increase his knowledge about a particular system, simulating his model, and thus increasing his information level. Here, activity measures the number of calculations achievable by the machine. The modeler has to adapt the available computational resources to the level of information he wants to obtain.

\subsubsection{Mathematical Model}

The modeler tries to obtain information of various types on a time interval $[0 ; T]$. Let be $I_{i t}$ the information of type $i=\{1, \ldots ; n\}$ available to the modeler in $t \in[0 ; T]$. We assume that model's value increases with the levels of various types of information available: $V\left(I_{1 t}, \ldots, I_{n t}\right)$. The value function is then

$$
V\left(I_{1 t}, \ldots, I_{n t}\right)=\sum_{i=1}^{n} f_{i}\left(I_{i t}\right)
$$

To choose a particular, and convenient form of function $f_{i}$, we assume now that the rate of value increase is decreasing with time. So the value function 
$V\left(I_{1 t}, \ldots, I_{n t}\right)$ regarding the various levels of information is strictly concave. This can be explained by the fact that for large amounts of information a further refinement of the information provides little value to the model. Moreover, beyond a certain "saturation threshold" for a certain type of information, we consider that it becomes difficult to treat this additional information leading to value decrease. Therefore, we obtain:

$$
V\left(I_{1 t}, \ldots, I_{n t}\right)=\Sigma_{i=1}^{n}\left(\beta_{i} I_{i t}-\frac{\alpha_{i}}{2} I_{i t}^{2}\right)
$$

With $\beta_{i} \in \mathbb{R}^{*,+}$ and $\alpha_{i} \in \mathbb{R}^{*,+}$ (the set of positive real numbers including 0 ). We notice immediately that $\frac{\partial V(\cdot)}{\partial I_{i t}}=\beta_{i} I_{i t}-\alpha_{i} I_{i t} \geq 0 \Leftrightarrow \widehat{I_{i}} \equiv \frac{\beta_{i}}{\alpha_{i}}$. So, it is obvious that the modeler never wants to obtain a level of information $i$ greater than $\widehat{I}_{i}$. Parameters $\beta_{i}>0$ and $\alpha_{i}>0$ reflect the fact that, for a given level of information, an increase of the level of a certain type of information does not generally have the same effect as an increase of the same level of an another type of information.

Activity measures the number of calculations achieved by the machine, over a period of time, in order to increase $I_{i t}$. Obtaining information of type $i$ requires the allocation of a certain amount of activity $P_{i t}$. Allocating activity between the augmentations of various types of information, the modeler will take into account that $0 \leq \sum_{i=1}^{n} P_{i t} \leq \bar{P}$, where $\bar{P}$ represents the maximal capacity of the computer.

The level of information of type $i$ increases with $P_{i t}$ but at a decreasing rate. So, the evolution equation of the information $i$ is :

$$
\dot{I}_{i}=f_{i}\left(P_{i t}\right)
$$

Where $f_{i}\left(P_{i t}\right)$ is a strictly concave function of $P_{i t}$. More specifically, we assume that $^{5}$ :

$$
\dot{I}_{i}=\gamma_{i} P_{i t}-\frac{\rho_{i}}{2} P_{i t}^{2}
$$

Where, $\gamma_{i} \in \mathbb{R}^{*,+}$ and $\rho_{i} \in \mathbb{R}^{*,+}$ reflect the differences between the various fact functions $f_{i}\left(P_{i t}\right)$.

Finally, the modeler's maximization program is:

$$
\max _{P_{i t} ; t \in[0 ; T] ; i=\{1, \ldots, n\}} \int_{0}^{T} \Sigma_{i=1}^{n}\left(\beta_{i} I_{i t}-\frac{\alpha_{i}}{2} I_{i t}^{2}\right) d t
$$

\footnotetext{
${ }^{5}$ We conventionally designed by an upper dot the derivative of a variable regarding time. Here $\dot{I}_{i}=\frac{d I}{d t}$. Furthermore the resolution of Equation 4 ensures that the acceptable range of the various activity values for the modeler is such that $f_{i}^{\prime}\left(P_{i t}\right) \geq 0$.
} 


$$
\begin{gathered}
\dot{I}_{i}=\gamma_{i} P_{i t}-\frac{\rho_{i}}{2} P_{i t}^{2} ; i=\{1, \ldots, n\} \\
I_{i 0}=0 ; I_{i T} \text { free; } i=\{1, \ldots, n\} \\
P_{i t} \geq 0 ; i=\{1, \ldots, n\} ; \forall t \in[0 ; T] \\
\Sigma_{i=1}^{n} P_{i t} \leq \bar{P} ; \forall t \in[0 ; T] \\
\widehat{I_{i}}-I_{i t} \geq 0 ; i=\{1, \ldots, n\} ; \forall t \in[0 ; T]
\end{gathered}
$$

Let $\lambda_{i t}$ be the covariable of $I_{i t}$, the Hamiltonian function of program 4 is:

$\mathcal{H}\left(I_{1 t}, \ldots, I_{n t}, P_{1 t}, \ldots, P_{n t}, \lambda_{1 t}, \ldots, \lambda_{n t}\right)=\sum_{i=1}^{n}\left(\beta_{i} I_{i t}-\frac{\alpha_{i}}{2} I_{i t}^{2}\right)+\sum_{i=1}^{n} \lambda_{i}\left(\gamma_{i} P_{i t}-\frac{\rho_{i}}{2} P_{i t}^{2}\right)$

In order to take into account the threshold of the various type of information and the constraints on the levels of activity we introduce the following the Lagrangean function:

$\mathcal{L}\left(I_{1 t}, \ldots, I_{n t}, P_{1 t}, \ldots, P_{n t}, \lambda_{1 t}, \ldots, \lambda_{n t}, \sigma_{t}, \mu_{1 t}, \ldots, \mu_{n t}\right)=\mathcal{H}()+.\sigma_{t}\left(\bar{P}-\sum_{i=1}^{n} P_{i t}\right)+\sum_{i=1}^{n} \mu_{i}\left(\widehat{I}_{i}-I_{i t}\right)$

Maximum principle leads to the following resolution conditions ${ }^{6}$ :

- Static optimality conditions:

$$
\begin{gathered}
\frac{\partial \mathcal{L}(\cdot)}{\partial P_{i t}}=\lambda_{i}\left(\gamma_{i} P_{i t}-\rho_{i} P_{i t}\right)-\sigma_{t} \leq 0 ; P_{i t} \geq 0 \text { and } P_{i t}=\frac{\partial \mathcal{H}(\cdot)}{\partial P_{i t}}=0 ; i=\{1, \ldots, n\} ; \forall t \in[0 ; T] \\
\frac{\partial \mathcal{L}(\cdot)}{\partial \sigma_{t}}=\bar{P}-\Sigma_{i=1}^{n} P_{i t} \geq 0 ; \sigma_{t} \geq 0 \text { and } \sigma_{t} \frac{\partial \mathcal{L}(\cdot)}{\partial \sigma_{t}}=0 \\
\frac{\partial \mathcal{L}(\cdot)}{\partial \mu_{i t}}=\widehat{I}_{i}-I_{i t} \geq 0 ; \mu_{i t} \geq 0 \text { and } \mu_{i t} \frac{\partial \mathcal{L}(\cdot)}{\partial \mu_{i t}}=0
\end{gathered}
$$

- Evolution equations of the covariables ${ }^{7}$ :

$$
\dot{\lambda}_{i t}=-\frac{\partial \mathcal{L}(\cdot)}{\partial I_{i t}}=\alpha_{i} I_{i t}-\beta_{i}-\mu_{i t}
$$

- Evolution equations of the information stocks:

\footnotetext{
${ }^{6}$ These conditions are necessary and sufficient because of the strict concavity of the Lagrangean function regarding the activity levels and the information levels.

${ }^{7}$ Whenever $\dot{\lambda}_{i t}$ exists, that means until $\widehat{I}_{i}$ is reached.
} 


$$
\dot{I}_{i}=\frac{\partial \mathcal{L}(\cdot)}{\partial \lambda_{i t}}=\gamma_{i} P_{i t}-\frac{\rho_{i}}{2} P_{i t}^{2}
$$

- Transversality conditions:

$$
\lambda_{i T}=0 ; i=\{1, \ldots, n\}
$$

- Conditions at the junction points $\tau^{8}$ :

$$
\lambda_{i \tau^{-}}-\lambda_{i \tau^{+}}=\pi(\tau) \frac{\partial\left(\widehat{I}_{i}-I_{i t}\right)}{\partial I_{i t}}=-\pi(\tau) ; \pi(\tau) \geq 0 \operatorname{and} \pi(\tau)\left(\widehat{I}_{i}-I_{i t}\right)=0
$$

Remembering that the costate variable of a stock measures the imputed value of this stock at any instant along the optimal path ${ }^{9}$, conditions 12 can be explained as follows: Since the modeler is free to choose the terminal value of a particular information stock, this value has to be such that his marginal contribution to model's value is zero at the end of the planning horizon.

Conditions 7-9 and 11 imply two possible cases regarding activity's allocation at instant $t \in[0 ; T]$ :

- Let be $j=\{1 ; \ldots ; m\}$; with $m \leq n$; the information level for which $I_{i t} \leq \widehat{I}_{i}$ at $t$. Assume first that $\sum_{j=1}^{n} \frac{\gamma_{j}}{\rho_{j}} \leq \bar{P}$. In this case, conditions 7-9 permit to conclude that:

$$
\begin{gathered}
P_{j t}=\frac{\gamma_{j}}{\rho_{j}} \equiv \widehat{P_{j}} ; \forall j=\{1, \ldots, m\} \\
P_{k t}=0 ; \forall k=\{m+1, \ldots, n\} \\
\sigma_{t}=0
\end{gathered}
$$

- Instead if $\sum_{j=1}^{n} \frac{\gamma_{j}}{\rho_{j}}>\bar{P}$ implies that $P_{j t} \leq \widehat{P_{j}} ; \forall j=\{1 ; \ldots ; m\}$. Same conditions as before lead to:

$$
\begin{gathered}
\lambda_{j t}\left(\gamma_{j}-\rho_{j} P_{j t}\right)=\sigma_{t} ; \forall j=\{1, \ldots, m\} \\
P_{k t}=0 ; \forall k=\{m+1, \ldots, n\}
\end{gathered}
$$

${ }^{8}$ Cf. [35], Possible junction points are the ones for which a value $\widehat{I_{i}}$ is reached and the terminal time $T$. Indeed this junction points are characterized by jumps in costate variables.

${ }^{9} \mathrm{Cf}$. [35]. For this reason, costate variables are called shadow prices in Economics. 
Equation 17 leads to a repartion rule of total activity available between the various $P_{j t}$. Indeed, for a particular $j$, this equation permits to obtain:

$$
\lambda_{j t}\left(\gamma_{j}-\rho_{j} P_{j t}\right)=\lambda_{l t}\left(\gamma_{l}-\rho_{l} P_{l t}\right) ; \forall l=\{1, \ldots, m\} ; l \neq j
$$

19 can be rewritten:

$$
P_{l t}=\frac{\gamma_{l}}{\rho_{l}}+\frac{\lambda_{j t}\left(\rho_{j} P_{j t}-\gamma_{j}\right)}{\rho_{l} \lambda_{l t}} ; \forall l=\{1, \ldots, m\} ; l \neq j
$$

So, using 8 :

$$
P_{l t}=\frac{\bar{P} \sum_{i=1}^{n} \rho_{l} \lambda_{l t}-\sum_{l=1}^{m}\left(\gamma_{l} \lambda_{l t}+\gamma_{l} \lambda_{l t}\right)}{\sum_{i=1}^{n} \rho_{i} \lambda_{i t}} ; \forall j=\{1, \ldots, m\} ; l \neq j
$$

Although this model was deliberately simplified, its resolution is made difficult because of cross-impact activity levels $(c f .21)$ and the possible presence of junction points.

\subsubsection{Model Resolution for a Single Information Type}

The model is resolved here for a single information type. Notations are similar to the general case, except for the omission of indexes $i$ used to design the various types of information. Since the objective function is an increasing function of $I_{t}$ and because $I_{0}=0$ the scope of the modeler is to reach the maximum amount of information $I_{t}$ using $P_{t}=\widehat{P}$ up to the possible point of time $\tau \in[0 ; T]$, where $I_{\tau}=\widehat{I}$.

Two cases are possible regarding the relative values of $\widehat{P}$ and $\bar{P}$ :

1. $\widehat{P} \leq \bar{P}$ : In this case the modeler chooses the activity level $\widehat{P}$ until $\widehat{I}$ is reached if it possible on $[0 ; T]$. Time $\theta$ for which $\widehat{I}$ is reached can be determine using 11 and the initial condition on $I_{t}$. Indeed, on $t \in[0 ; \theta]$ we can rewrite $: \dot{I}=\gamma \widehat{P}-\frac{\rho}{2} \widehat{P}^{2} \Rightarrow I_{t}=\left(\gamma \widehat{P}-\frac{\rho}{2} \widehat{P}^{2}\right) t$. So $\theta=\frac{2 \widehat{I}}{2 \gamma \widehat{P}-\rho \widehat{P}^{2}}$. So, on $[0 ; T]$, two situations are possible:

- If $\theta \leq T$; the modeler chooses $P_{t}=\left\{\begin{array}{c}\widehat{P} ; \forall t \in[0 ; \theta] \\ 0 ; \forall t \in] \theta ; T]\end{array}\right.$ and $I_{t}=$ $\left\{\begin{array}{c}\left(\gamma \widehat{P}-\frac{\rho}{2} \widehat{P}^{2}\right) t ; \forall t \in[0 ; \theta] \\ \widehat{I} ; \forall t \in] \theta ; T]\end{array}\right.$. The corresponding temporal path is a line starting at point $(0 ; \widehat{P})$ and ending at $(\widehat{I} ; \widehat{P})$, in exactly $\theta$ periods. 
- If $\theta>T$; the modeler chooses $P_{t}=\widehat{P} ; \forall t \in[0 ; T]$ and $I_{t}=(\gamma \widehat{P}-$ $\left.\frac{\rho}{2} \widehat{P}^{2}\right) t ; \forall t \in[0 ; T]$. So the terminal value of the stock is $I_{T}=(\gamma \widehat{P}-$ $\left.\frac{\rho}{2} \widehat{P}^{2}\right) T$. The corresponding temporal path is a line starting at point $(0 ; \widehat{P})$ and ending at $\left(I_{T} ; \widehat{P}\right)$, in exactly $T$ periods.

2. $\widehat{P} \geq \bar{P}$ : In this case the modeler chooses the activity level $\bar{P}$ until $\widehat{I}$ is reached if it is possible on $[0 ; T]$. Using the same notation as before, two situations are possible:

- If $\theta \leq T$; the modeler chooses $P_{t}=\left\{\begin{array}{c}\bar{P} ; \forall t \in[0 ; \theta] \\ 0 ; \forall t \in] \theta ; T]\end{array}\right.$ and $I_{t}=$ $\left\{\begin{array}{c}\left(\gamma \bar{P}-\frac{\rho}{2} \bar{P}^{2}\right) t ; \forall t \in[0 ; \theta] . \\ \widehat{I} ; \forall t \in] \theta ; T]\end{array}\right.$. The corresponding temporal path is a line starting at point $(0 ; \bar{P})$ and ending at $(\widehat{I} ; \bar{P})$, in exactly $\theta$ periods.

- If $\theta>T$; the modeler chooses $P_{t}=\bar{P} ; \forall t \in[0 ; T]$ and $I_{t}=(\gamma \bar{P}-$ $\left.\frac{\rho}{2} \bar{P}^{2}\right) t ; \forall t \in[0 ; T]$. So the terminal value of the stock is $\bar{I}_{T}=(\gamma \bar{P}-$ $\left.\frac{\rho}{2} \bar{P}^{2}\right) T$. The corresponding temporal path is a line starting at point $(0 ; \bar{P})$ and ending at $\left(\bar{I}_{T} ; \bar{P}\right)$, in exactly $T$ periods.

\subsection{Activity Definition from an Economics Point of View}

Activity has been investigated both in the general Economics framework and using one major modeling approach used in Economics: Optimal control theory. We can conclude the following definition of activity:

- What is activity in this context? Any phenomenon impacting a scarce resource.

- Why use it? Because this is the link between core economic concepts: Classical economics gives the activities result (measured with utilities and global welfare). Behavioral economics explains why activities are selected. Activity analysis could describe where and when events occur. It can give a description of the spatiality and of the dynamicity of an economic system.

- How to define it? The number of events concerning scarce ressources.

- Where to use it? With any economic model where economic events occur at different time/locations.

- When to use it? When anyone wants to know what people do, where and when they do it, not only why they do it and how happy they are. 


\section{A Prospective Approach: Activity Framework in Models of Human Beliefs and Knowledge}

This last section mainly is prospective. Its function is to show that concepts such as activity tracking and activity awareness are unifying ones and that they are particularly promising when applied to the models of human beliefs and knowledge.

\subsection{A New Paradigm in Cognitive Sciences and in the Psychology of Reasoning}

In contemporary cognitive science and psychology of reasoning, the construction of beliefs and knowledge is largely seen as relying on complex and heterogeneous processes.

Nevertheless, although models of such processes often differ to some large extent in the literature, many researchers belonging to different disciplines (psychology, AI, epistemology), more and more agree on the fact that, when reasoning, human minds do not follow the classical models of rationality, be they of a probabilistic nature (such as the classical models of inference: see the critics in [36, 37] or of a deductive and logical nature (such as the classical models of belief revision: see the critics in [38][39])).

Following some seminal ideas of [40] and, first of all, grounding their arguments on a significant amount of new empirical evidences especially since the 1980 's, they put at the forefront the hypothesis that human reasoning is a resource-limited, context-sensitive, time-consuming, non-optimal and sometimes non-terminating process $[41,37,38,17]$.

For the viewpoint of a general and conceptual framework based on activity tracking and activity awareness, it is crucial to notice that this new paradigm largely stems from a new sensibility of researchers to the property of cognition and reasoning to be situated, only locally (in time and space) optimal and goaloriented activities, just as are many of our other activities.

\subsection{Concepts Already Related to Activity in Cognitive Sciences}

Grounding our conceptual and prospective argument on such a consensual paradigm, it is reasonable to assume that the best models of the construction of beliefs and knowledge we can build today are 1) component-based ones in the sense given in section 1 of this paper, 2) activity oriented in that the focus is no more on formal and uninterpreted deductive symbols and on their syntactic links (such as in the abstract logical models of reasoning dating back to the first symbolic AI 
of the 50's) but on the various, simultaneous and concurrent kinds of cognitive and emotional activities operating in an overall process of reasoning.

It is important to discern that, at this general level of an activity oriented conceptual framework, what we call a component-based approach does not necessarily entail any strong, nor massive nor weak modular vision of cognition, in the sense of Fodor [42] or even [43] or of Carruthers [44] for instance ${ }^{10}$. There is a strong methodological advantage in our grounding the conceptual analysis not on modules but on activities: It gives the possibility to consider such or such thesis or competing argument or algorithm in a process of belief revision, for instance, as a real competing component of the overall model. Hence, when we speak of components, no ontological commitment to real cognitive modules is necessary.

In the following paragraphs, we will present a sample of some more precise concepts that specifically can be interpreted in terms of activity tracking and activity awareness.

First, such an interpretation seems possible for the models of inference. Whereas practical cognition intervenes in the process of decision making, epistemic cognition intervenes in the process of the justification of beliefs [46, 38]. Ordinarily, due chiefly to time limitations, human minds seem to operate in a way that both minimizes and optimizes their activity in some given context and facing some given concern, be it practical or epistemic. From these considerations, it seems reasonable to assume that "concise and efficient structures" [47] emerge or exist in the mind - and/or in the brain: see [48] - and play a major role both in practical and epistemic cognition.

At another level, in the specific domain of the philosophy of science, similar ideas have arisen. E.g., [49, 50] claims that a scientist does not operate as a single autonomous information processor and that his or her work cannot be reduced to a purely logical construction. According to him, the scientist always belongs to a social network. As a node of this network, he or she co-operates in a huge distributed information processing. Hence, from this socio-psychological viewpoint on science, the collective construction of knowledge has to solve some problem of optimizing the activity (intensity of interaction) between actors.

Finally, in the domain of cognitive science, the question of the knowledge and/or the simulation and anticipation of the contents of the minds of others, frequently called the mind-reading question, has led many to build different and concurrent models of activity awareness in the mind.

Facing these new trends in psychology of reasoning, epistemology and cognitive science, our claim is that single human minds or human minds in networks can, to some extent, be compared to some systems of computation trying to optimize either their activity or their awareness of the activity of others - or of themselves - so as to decrease their execution time. Hence, the aim of this

${ }^{10}$ See [45] for a review on modularity in cognitive sciences. 
section on activity models of beliefs and knowledge is to illustrate to what extent the concepts of activity, activity tracking, activity awareness, engineering phase, modeling phase and decision phase could be well-adapted to the development of the ideas and models in these four domains of epistemology: inference, belief revision, scientific discovery and mind-reading.

\subsection{Activity in Models of Inference: Reasoning the Frugal Way}

The model of inference presented by [36] consists of simple cognitive algorithms which suggest cues and performs inference from memory. As such, "it uses limited knowledge as input" (ibid.). To compensate for this limitation, such models assume that inductive inference has to take into account the natural environment of the cognitive agent. In this case, the environment is implemented in his memory. Although the algorithm is not linear and does not use all available information (it violates the traditional standards of rationality), it leads to remarkable results compared to other classical models. Such algorithm is designed to enable quick decision making. In this work, the model is multicomponent in that it is composed of probabilistic atomic mental models: but the decomposition can be deepened or flattened.

As their success is remarkable, we propose that such algorithms be considered less arbitrary than others. They can be taken as examples of more realistic simplifications of a computational procedure thanks to an "activity tracking" process applied to some other, less performing, hence more fictive, classical models of inference.

- What is activity in this context? The activity of the coupled model is the sum of the numbers of internal and external events of atomic models, over a simulation time period

- What is optimized activity in this context? It is a realistic and effective psychological mechanism (such as the simple algorithms of [36]) which would appear quite different from the fictive computational processes as they are hypothesized in classical inference models purely based on deduction and logic. Optimized activity is the most efficient (the quickest and the most successful) algorithmic process that leads to a certain cognitive goal: in this sense, it is the one that is the most efficient relatively to the goal and compared to other computational models, whereas these other models lead to useless and time-consuming (inactive in this sense) computations. Accordingly, we can make the heuristic hypothesis that activity is what really happens in the mind, hence, in the brain.

- Why use it? 1) To optimize the rapidity and the efficiency of the models of inference and decision-making (both in epistemic and practical cognition);

2 ) To build more realistic and more reusable models of the human inference 
(taking time but not too much, based on limited-resources, highly sensitive to contexts,...)

- Where to use it? In AI in general, in decision-making, in the modeling of practical cognition or common sense reasoning, to ameliorate the efficiency of that kind of complex systems mixing computer-aided decision-making and/or augmented-reality (planes).

- When to use it? We use it when we are sure to possess a simplifying model of inference for a precise kind of task.

\subsection{Activity in Beliefs Revision: Active Cycles of Defea- sible Reasoning}

In his procedural epistemology, [38]suggests viewing the process of belief justification as a dynamic, evolving, sometimes non-terminating, process. Its core hypothesis is that, most of the time, the human mind has not enough resource to compute all the implications some new candidate belief would have in the set of the already accepted (because to some extent already justified) beliefs to be able to genuinely warrant or reject it. This is the reason why most of our justified beliefs are not completely warrantable. They are said to be "defeasable": at any time, during our life, i.e. during our new interactions with others and with the environment, most of our beliefs can be replaced by their contrary or by other different beliefs.

"In particular, beliefs can be defeated by further reasoning, and may later be reinstated by defeating their defeaters. So beliefs may cycle in and out of the set of justified beliefs many times as reasoning progresses. A belief is only warranted if the cycling eventually stops and the belief subsequently remains justified" ([38], 20-21 .)

We propose to design this cycling as analogous to activities performed by the mind within its set of beliefs. A belief is warranted or rejected when its justification status no longer changes.

From a component-based and activity oriented viewpoint, it can be suggested viewing beliefs as atomic models having autonomous activity. A given belief model is set to the state "warranted" if one condition of implication is received and if no condition of rejection has been sent to it. Each belief model interacts with others by sending to all others its epistemic implications, which serves to warrant other beliefs, and its own condition of warranty so as to verify whether it still is coherent or not with other already warranted beliefs. When two beliefs appear to be incompatible, defeasibility has to occur, either for the one or for the other. Thereafter, different strategies of choice for defeasability can be tested: hence optimal strategies of constructing systems of beliefs can be sought through computational experiments. 
- What is activity in this context? Activity is the total number of internal changes of beliefs, only, if the internal state of a given belief does not change there is no activity in that the content of the model corresponding to the cognitive content of the mind has not changed (even if this belief still permanently interacts with other beliefs so as to test its compatibility).

- What is an optimized activity in this context? Activity is the core of the reasoning process of the mind through which it permanently tries to establish its belief. A mind is all the more active, from this viewpoint, as it is subject to doubts and revision of its beliefs. From this viewpoint, if activity of the mind is desired, an optimized model can mean a maximization of activity, not a minimization.

- Why use it? Such vigilance has often been compared to one of the characteristics of the consciousness: versatility, capability to deliberate and choose. Activity tracking in the context of belief revision could be similar to some intentional states tracking.

- How to define it? Activity could be defined as the measure of the cognitive reactivity or cognitive attention and vigilance of the mind in a given context. Where to use it? In AI, in cognitive psychology, in the modeling of reasoning, in the modeling of the dynamics of intentional states of the mind.

- When to use it? When we aim at designing an efficient and rapid model of decision-making, or when we aim at understanding the process of practical and epistemic cognition.

\subsection{Activity in Netcentric Social Studies of Science}

According to [49], "Socially and cognitively, science involves a tension between cooperation and competition, and researchers are only beginning to understand how social organization can contribute to the overall goal of increasing scientific knowledge [...] By combining a computational understanding of individual cognition with an analysis of scientific communities in terms of distributed computation, we can start to see how sociological and psychological accounts of science can be integrated." More specifically, as recalled by Thagard $[49,50]$ or even by [51], in this perspective of a computational philosophy of science, it is desirable to design computational experiments to test the efficiency of various strategies in the process of the construction of scientific knowledge. As any other kinds of distributed computation, a given social strategy and social dynamics for science can be evaluated in terms of activity and activity tracking. The components or nodes of such a coupled model can be the models of the actors that are cooperating and competing: those actors are the scientists themselves or the academic institutions they belong to. 
- What is activity in this context? The intensity or frequency of the information exchanges between nodes or clusters of nodes, in a given region of the social network.

- Why use it? It could be a good criterion for evaluating the fruitfulness of a given strategy of interactions and exchanges between actors.

- How to define it? It is the rate and intensity of the use of a given link between certain nodes in the context of a given strategy.

- Where to use it? To design and evaluate social strategies of science (policy of science, knowledge management), to help to understand some discoveries in some periods of history of science.

- When to use it? When we are sure to have enough data about the network and about the social and individual strategies. This restriction can be a bottleneck. Such optimized models of social networks occurring in the construction of science can be used in historical studies but also in prospective studies, especially in those concerning the policy and management of scientific research.

\subsection{Activity in the Models of Mind-reading}

After having presented a model of mind-reading based on the concepts of control theory where the "social interaction loop" is internally emulated in a cross-modal emulator (i.e. a coupling between an egocentric emulator and an allocentric emulator, the second one representing the situation, beliefs, intentions and actions of others) [52] concedes that:

"It is clear that when modeling the higher levels of inter-subjectivity with the aid of control theory, methodological issues become pressing. The number of variables and the level of complexity will be so high that it will become difficult to evaluate a model." (235)

Of course, choosing control theory to model mind-reading is consistent with the Gärdenfors' geometrical and topological theory of conceptual spaces [53]. But it is significant that when we shift to an alternative theory which focuses on the massive modularity of mind - such as Carruthers' - this kind of "self-monitoring module" [44] becomes less convincing. Carruthers argues that:

"Naive subjects are bad at reasoning about reasoning - at identifying mistakes in reasoning, at theorizing about standards of good reasoning, and at improving their own and other's reasoning." (ibid: 184).

This is the reason why, according to him, even in the case of mind-reading, most people use heuristic ways of reasoning similar to the ones experimented by [36]. 
- What is activity in this context? Activity is the total number of events occurring in the coupled model (the allocentric atomic model + the egocentric atomic model) over a simulation time period. If we adopt Gärdenfors' view, activity awareness is optimal when the optimal control due to a good choice of the parameters of the allocentric emulator is reached. If we adopt Carruthers's view, in the context of the mind-reading question, we are invited to adapt the heuristic models of activity tracking by [36] in terms of activity awareness.

- Why use it? It could lead models of mind-reading to more direct confrontation with experimental data as in [54]. For instance, it could help to choose between a massive modularity approach (Carruthers) or a topological one (Gärdenfors).

- How to define it? Activity appears here in a context of activity awareness, similarly to the role of this same concept in the context of collaborative software. Activity awareness measures the degree of coincidence - or of efficiency - of a model of other's mind seen from the viewpoint of a given mind.

- Where to use it? In cognitive sciences. Where it is desirable to enhance our theory of the reasoning or of the reacting of others: in strategy problems, for the modeling of collaborative work, for the modeling of endomorphic agents [55].

- When to use it? In general, activity awareness is useful when a kind of optimal or "frugal" awareness is assumed to operate in a given faculty of the mind.

\subsection{Activity in the Reasons of Beliefs and Activity Aware- ness in Cognition}

In his sociological theory of beliefs, [56] has shown that human minds often have what he calls "good reasons" to believe what they believe. A "good reason" is a reason that leads an agent to a conclusion which he cannot induce through any other nor more reachable reason, but which is functionally (biologically, psychologically and/or socially) advantageous for him, although he still can have some doubts about the rational legitimacy of this "reason".

Let's assume that a system can survive only if its resource expenditure allows it to, i.e. only if it is functional in this broad sense. The survival of the system is decided through a benefit/cost ratio which has to be greater than 1 . In every context, it is possible to define the "benefit" of the system as the resources acquired and its "cost" as the resources expended. Activity can be defined as the number of transitions of the model of the system. Hence, activity can be a measure of the resources expended. 
So, the activity awareness of the model of a cognitive system can be used in a module of auto-evaluation operating at runtime: through that, the model of the cognitive system can evaluate the achievement of its cognition while cognizing, and make itself evolve the optimal way. In other words, this principle of activity awareness could enhance many of the already existing techniques that are used in auto-adaptive simulations of cognition.

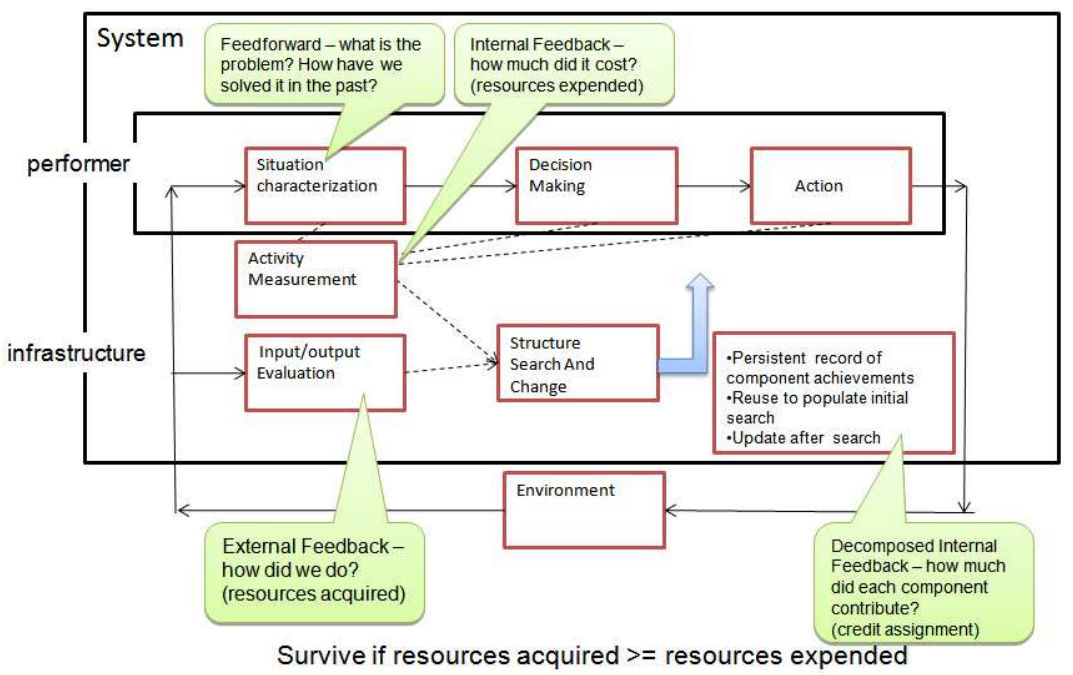

Figure 10: Activity-Aware System Architecture.

From this focus on the self-optimization of a cognitive system through activity awareness, it follows that, in each context, we will have to complete the list of our questions and ask what is the kind of benefit (the resource) that can be acquired.

1. In the case of models of inference, the evolution will lead to frugal models and the resources acquired will be of a biological (neurological) nature.

2. In the case of belief revision, the model of the system cannot waste too many resources in reconciling inconsistent beliefs. So it will have to evolve toward systems that do only enough belief revision to enable benefit/cost ratios $>=1$, i.e., this in an infrastructure, so just enough to enable the consistency of beliefs to support frugal decision making. In this context, the resources acquired is indirect through support of decision making.

3 . In the context of social network, the benefit can be considered at 2 distinct levels: at the network level, this system cannot strain communication 
resources beyond satisficing benefit/cost ratio; at individual level, this system cannot strain communication resources beyond satisficing benefit/cost ratio. But it is not always clear how these ratios are manifested. E.g., how large can a social network grow?

4. In the context of mind reading, this functionality requires high expenditure of mental resources. Moreover, the kind of benefit can change according to the kind of mental capacity modeled. E.g. the kind of benefit could be to have acquired the ability not to be outwitted by adversary, but not only (it could be the ability to be easily mind read or not by other minds, etc.). Nevertheless, a challenge could be to search for a Universal Cognitive Modeler that could optimize many different mental capacities at the same time.

Finally, it appears that the kinds of benefit could change in each context but not the principles which are at the basis of such an Optimal Activity Aware System Architecture. Most of all, the modules of "Activity measurement" and "Structure Change", as it is seeable in Figure 10, will be central for a sufficiently rich evaluation of the contributions of components taking place in the "Internal feedback" module.

\subsection{Life Cycle of Simulation in Activity-based Models of Beliefs and Knowledge}

As we can now understand, first of all, such models of beliefs and knowledge traditionally tend to belong to the "Modeling phase" as it is presented in section 1: The goal of the psychologists of reasoning or of the epistemologists is to model and to explain a given psychological task with some validated model.

But, in a second step, as it appears too in most of the works we have quoted, and as it is explicitly shown in the work of [36], the psychologists or epistemologists have to look for concurrent models that, to be realistic, have to minimize execution times and even maximize reusability. As such, their task is analogous to that of the software engineers in their "Engineering phase": they have to look for some optimized models too. Subsequently, as they take into account the limited resources of human mind, such optimized models can be seen as good candidates for more realistic representations of what the human brain really does.

Finally, at the end of the simulation cycle, these more realistic models of beliefs and knowledge construction can be used for some real applications in artificial intelligence. In this context and for this aim, psychologists will have to choose the most adapted model among the already efficient and realistic models: so, they will enter a "Decision-Maker phase" too.

Indeed, we can notice that, in principle, it remains not necessary to use a realistic model to perform some intelligent task. Even if it is a good methodological 
principle in AI, we do not have to assume that nature already has explored and selected the most efficient heuristics for the construction of reasonings and beliefs. Analogously, modelers of reasoning can, to some extent, explore some direct link between phase 2 and phase 3 , without always having to ground their models first on realism and on the explanation of some strategies of reasoning already existing in nature (phase 1).

\section{Conclusion}

According to the conceptual tools used in each discipline, different points of view on activity emerged based on definitions provided in Section 2:

- In Section 3: Activity concept is used in a cellular automata structure, activity consists of two levels:

- Activity in cells: Activity corresponds to a state change in a cell.

- Activity in configurations: An activity configuration consists of active and inactive cells.

- In Section 4: A biological characterization of activity concept, inspired from chemistry, corresponds to a measure of exchanges (gas, liquids, nutrients, molecules...) and of transformations (chemical transformations) which result in maintaining the integrity of the object itself or the system which it belongs.

- In Section 5: From an economical point of view, activity concept relates to agent rationality when affecting scarce resources. It is a measure and a modeling of sequences of rules activation.

- In Section 6: An epistemological consideration of activity considered, from a human-centric perspective, the following possible multilevel usages:

- At the inference level: Activity concept is an abstraction of mind activity. Activity concept corresponds to a ranking of the most efficient (active) algorithms emulating decisions.

- At the beliefs revision level: Activity concept is a measure of mind activity, in doubts and revisions of beliefs.

- At the mind reading level: Activity concept is an evaluation of the efficiency of a model of other's mind by a given mind.

- At the net centric social level: Activity concept is the frequency of information (ex)changes in/between nodes of a region in a social network.

- At an awareness level: Activity concept is an auto-evaluation of cognition achievement, through a ratio of resources expanded/acquired. 
A three level hierarchy can be built as an architecture for constructing componentbased systems:

1. Activity tracking: Tracking activity, at component or configuration levels, allows optimizing simulator resources. This first layer can be considered as a first physical/biological (Sections 4 \& 5), material, characterization of simulation models. Automatic detection mechanisms reduce execution times. Analyzing basic changes in activity can also enhance model understanding (cf. Section 4).

2. Activity evaluation: Using the built-in detection mechanisms of level 1 , activity can be measured as the fractional time that a component contributes (Section 6). Correlating contribution with outcome, a credit can be attributed to components. Therefore, a real measure of performance of components, in a context, can be achieved. This allows for automatic composition and re-use of components. In social sciences, a direct analogy exists between activity evaluation and the evaluation of rules, actions, decisions... of agents (Sections 2 and 3 ).

3. Activity Awareness: Considering cognitive systems through the concepts of activity and activity tracking presents the advantage to let us see these systems from a dynamical point of view. A second advantage could be to use this dynamical viewpoint and representation to let the system find itself its optimal design regarding its resource expenditure (assuming that some resource expenditure is always linked to the type of activity at stake.) The activity awareness of the model of a cognitive system can be used in a module of auto-evaluation operating at runtime (Section 6).

We believe that levels 1 to 3 constitute a cycle (activity awareness mechanisms can be re-implemented partially or totally at level 1 - component level as builtin mechanisms) .

\section{Acknowledgements}

This research is partially supported by the Centre national de la recherche scientifique (CNRS).

\section{References}

[1] S. R. Akerkar, Analysis and visualization of time-varying data using the concept of 'activity modeling', Master's thesis, Dept. of Electrical and computer engineering - University of Arizona (2004).

[2] K. Tocher, The art of Simulation, English University Press, 1963. 
[3] O. Balci, The implementation of four conceptual frameworks for simulation modeling in high-level languages, in: WSC '88: Proceedings of the 20th conference on Winter simulation, ACM, New York, NY, USA, 1988, pp. 287-295. doi:http://doi.acm.org/10.1145/318123.318204.

[4] M. Pidd, Object-orientation and three phase simulation, in: Proc. of Winter Simulation Conference, 1992, pp. 689-693.

[5] E. E. Catmull, A subdivision algorithm for computer display of curved surfaces, Ph.D. thesis, Dept. of CS, U. of Utah (Dec. 1974).

[6] J. Clark, Hierarchical geometric models for visible surface algorithms, Communications of the ACM 19 (10) (1976) 547-554.

[7] T. A. Funkhouser, C. H. Sequin, Adaptive display algorithm for interactive frame rates during visualisation of complex virtual environments, Proceedings of SIGGRAPH'93 (1993) 247-254.

[8] J. F. Ramos, M. Chover, Level of detail modelling in a computer game engine, in: M. Rauterberg (Ed.), ICEC, Vol. 3166 of Lecture Notes in Computer Science, Springer, 2004, pp. 451-454.

[9] P. Horn, Autonomic computing: IBM's perspective on the state of information technology, Manifesto, IBM Research (Oct. 2001).

[10] R. Sterritt, M. Parashar, H. Tianfield, R. Unland, A concise introduction to autonomic computing, Advanced Engineering Informatics 19 (3) (2005) 181-187.

URL http://dx.doi.org/10.1016/j.aei.2005.05.012

[11] M. Weiser, Ubiquitous computing (abstract), in: ACM Conference on Computer Science, 1994, p. 418.

URL http://doi.acm.org/10.1145/197530.197680

[12] W. E. Leland, T. J. Ott, Load balancing heuristics and process behavior, ACM Performance Evaluation Review 14 (1) (1986) 54-69.

[13] J.-L. Giavitto, C. Godin, O. Michel, P. Prusinkiewicz, Modelling and Simulation of biological processes in the context of genomics, Hermes, 2002, Ch. "Computational Models for Integrative and Developmental Biology". URL http://www.ibisc.fr/ michel/PUBLIS/2002/autran02.ps.gz

[14] P. Coquillard, D. Hill, Modélisation et Simulation des Ecosystèmes, Masson, 1997.

[15] J. von Neumann, Theory of Self-reproducing Automata, University of Illinois Press, 1966. 
[16] A. Muzy, D. Hill, What is new with the activity world view in modeling and simulation? using activity as a unifying guide for modeling and simulation, in: Winter Simulation Conference, Phoeninx, Arizona, Invited paper, 2011, pp. 2887-2899.

[17] C. Ivan-Chesnevar, A. Maguitman, R. Loui, Logical models of argument, ACM Comput. Surv. 32 (4) (2000) 337-383. doi:10.1145/371578.371581. URL http://portal . acm.org/citation.cfm?id=371581

[18] J. Buxton, J. Laski, Control and simulation language, The Computer Journal 5 (1962) 194-199.

[19] B. P. Zeigler, T. G. Kim, H. Praehofer, Theory of Modeling and Simulation, Academic Press, 2000.

[20] B. P. Zeigler, Theory of Modeling and Simulation, John Wiley, 1976.

[21] A. Muzy, R. Jammalamadaka, B. Zeigler, J. Nutaro, The activity tracking paradigm in discrete-event modeling and simulation: The case of spatially continuous distributed systems, Simulation Transactions of the Society for Modeling and Simulation International 5 (87) (1962) 449-464.

[22] A. Muzy, E. Innocenti, G. Wainer, A. Aiello, J. F. Santucci, Specification of discrete-event models for fire spreading, Simulation 81 (2) (2005) 103-117.

[23] G. Wainer and N. Giambiasi, Application of the cell-DEVS paradigm for cell spaces modeling and simulation, Simulation 76 (2001) 22-39.

[24] A. Muzy, J. Nutaro, Algorithms for efficient implementation of the devs and ds-devs abstract simulators, in: Open International Conference on Modeling and Simulation (OICMS'05), 2005, pp. 273-279.

[25] A. Muzy, E. Innocenti, A. Aiello, Dynamic Structure Cellular Automata in a Fire Spreading Application, in: Proc. of ICINCO 2004, Vol. 3, 2004, pp. $143-151$.

[26] P. Siregar, J. P. Sinteff, N. Julen, P. L. Beux, An interactive 3D anisotropic cellular automata model of the heart, Computers and Biomedical Research 31 (5) (1998) 323-347.

[27] R. W. Gosper, Exploiting regularities in large cellular spaces, Physica D: Nonlinear Phenomena 10 (1-2) (1984) 75-80.

[28] H. H. Chen, T. S. Huang, A survey of construction and manipulation of octrees, Computer Vision, Graphics, and Image Processing 43 (3) (1988) 409-431.

[29] D. P. Tans, D. R. Keeling, National oceanic and atmospheric administration (noaa) (2012).

URL www.esrl.noaa.gov/gmd/ccgg/trends/ 
[30] P. Coquillard, Simulation of the cyclical process of heathlands: Induction of mosaic structures, evolution to irreversible states, Ecological Modelling $80(2-3)(1995)$ 97-111.

[31] E. Malinvaud, Lectures on miroeconomic theory.

[32] G. S. Becker, The economic approach to human behavior, University of Chicago Press, 1976.

[33] H. A. Simon, From substantive to procedural rationality, Method and appraisal in economics (1980) 129.

[34] R. J. Aumann, Rule rationality vs. act rationality, The Hebrew University, Center for the Study of Rationality.

[35] D. Leonard, L. N. V., Optimal Control Theory and Static Optimization in Economics, Cambridge University Press, 1992.

[36] G. Gigerenzer, D. G. Goldstein, Reasoning the fast and frugal way: Models of bounded rationality, Psychological review 103 (4) (1996) 650-669.

[37] S. P. T. A. Kahneman, D., Judgment under uncertainty: Heuristics and Biases, Cambridge University Press, 2008.

[38] J. Pollock, Procedural epistemology, The Digital Phoenix - How computers are changing philosophy (1998) 17-36.

[39] J. Bonnefon, Le raisonneur et ses modèles, PUG, 2011.

[40] H. A. Simon, A behavioral model of rational choice, The quarterly journal of economics 69 (1) (1955) 99-118.

[41] A. Goldman, Epistemology and Cognitio, Harvard University Press, 1988.

[42] J. Fodor, Modularity of Mind: An Essay on Faculty Psychology, MIT Press, 1983.

[43] J. Fodor, The mind doesn't work that way: The scope and limits of computational psychology, MIT Press, 2000.

[44] P. Carruthers, The Architecture of the Mind, Oxford University Press, USA, 2006.

[45] H. Barrett, R. Kurzban, Modularity in cognition: Framing the debate, Psychological Review 113 (2002) 628-647.

[46] J. Pollock, Plans and decisions, Theory and Decision 57 (2005) 79-107.

[47] A. Muzy, B. P. Zeigler, Introduction to the activity tracking paradigm in Component-Based simulation, The Open Cybernetics and Systemics Journal 2 (2008) 48-56. 
[48] G. Tononi, A. R. McIntosh, D. P. Russell, G. M. Edelman, Functional clustering: Identifying strongly interactive brain regions in neuroimaging data, Neuroimage 7 (2) (1998) 133-149.

[49] P. Thagard, Computation and the philosophy of science, The Digital Phoenix - How computers are changing philosophy (1998) 48-61.

[50] P. Thagard, Societies of minds: Science as distributed computing, Studies in History and Philosophy of Science 24 (1993) 49-67.

[51] D. Dennett, Artificial life as philosophy, Artif. Life 1 (3) (1995) 291-292. URL http://portal.acm.org/citation. cfm?id=1667940

[52] P. Gärdenfors, Mind-reading as control theory, European Review 15 (02) (2007) 223-240.

[53] P. Gärdenfors, Conceptual spaces: the geometry of thought, MIT Press, 2000.

[54] M. Jeannerod, Motor cognition: What actions tell the self, Oxford University Press, USA, 2006.

[55] B. Zeigler, A. Muzy, L. Yilmaz, Artificial intelligence in modeling and simulation, in: Encyclopedia of Complexity and Systems Science, 2009, pp. $344-368$.

[56] R. Boudon, The art of self-persuasion : the social explanation of false beliefs / Raymond Boudon ; translated by Malcolm Slater, Polity, Cambridge, UK ; Cambridge, MA :, 1994, includes bibliographical references (p. [278]-312) and index. 